\title{
Bridging the Gap Between Nanophotonic Waveguide Circuits and Single Mode Optical Fibers Using Diffractive Grating Structures ${ }^{\star}$
}

\author{
G. Roelkens*, D. Vermeulen, F. Van Laere, S. Selvaraja, S. Scheerlinck, D. Taillaert, \\ W. Bogaerts, P. Dumon, D. Van Thourhout, and R. Baets \\ Photonics Research Group, Ghent University/IMEC, Sint-Pietersnieuwstraat 41, B-9000 Ghent, Belgium
}

\begin{abstract}
In this paper, the use of diffractive grating structures to efficiently interface between a single mode fiber and a high index contrast waveguide circuit is outlined. We show that high index contrast grating structures allow for broadband and high efficiency coupling. Since no polished facet is required on the photonic integrated circuit to interface with the optical fiber, fiber-to-chip grating couplers enable wafer-scale testing, reducing the cost for testing large scale integrated optical circuits. We show that two-dimensional grating structures can solve the problem of the huge polarization dependence of high index contrast photonic integrated circuits. Finally, an optical probe is presented, which allows testing individual components of a photonic integrated circuit, analogous to the electrical probes used in micro-electronics.
\end{abstract}

Keywords: Silicon Photonics, Fiber Interface.

\section{CONTENTS}

1. Introduction

2. Fiber-to-Chip Grating Coupler: Operation

Principle and Limitations .

3. Focusing Grating Couplers

4. Extending the Bandwidth: Grating Duplexer Structures

5. Two-Dimensional Grating Structures-A Polarization Diversity Scheme.

6. The Optical Probe

7. Conclusions ...

Acknowledgments .

References and Notes

\section{INTRODUCTION}

Nanophotonic integrated circuits allow large scale integration of optical functions on a single substrate. The routing of light on these ultra-compact circuits is achieved using high refractive index contrast waveguides, since wavelength scale optical functions can be realized in this way. In order for the waveguides to be single mode, submicron waveguide dimensions are required. The mismatch between the size of the optical mode on the photonic integrated circuit and that of a single mode fiber, used to

\footnotetext{
*Author to whom correspondence should be addressed.

${ }^{\star}$ This is an invited review paper.
}

interface with the photonic integrated circuit, is very large. Therefore a dedicated coupling structure between optical fiber and photonic integrated circuit is required for efficient fiber-chip coupling. Two main approaches are pursued to tackle this problem. In a first approach, the size of the optical mode on the chip is transformed using adiabatic taper structures. Both lateral inverted taper structures (where the optical mode is transformed to that of a low index waveguide structure on top of the nanophotonic waveguide layer $)^{1}$ and three dimensional taper structures are considered. Although in principal the transformed optical mode can be matched to that of a single mode fiber, this leads to long adiabatic taper structures and to a large topography on the photonic integrated circuit, which is unwanted from a fabrication point of view. Therefore the adiabatic taper approach is often combined with a decrease in the size of the fiber mode using lensed fiber or high numerical aperture fiber. While in this way high efficiency, broadband optical coupling can be realized, the adiabatic taper approach has some disadvantages. It typically requires the use of specialty optical fiber and/or specialty fabrication techniques. Moreover, it requires a polished facet to interface with the photonic integrated circuit. While this is the standard approach of interfacing with a photonic integrated circuit nowadays, it is desirable to have a wafer-scale approach when it comes to 

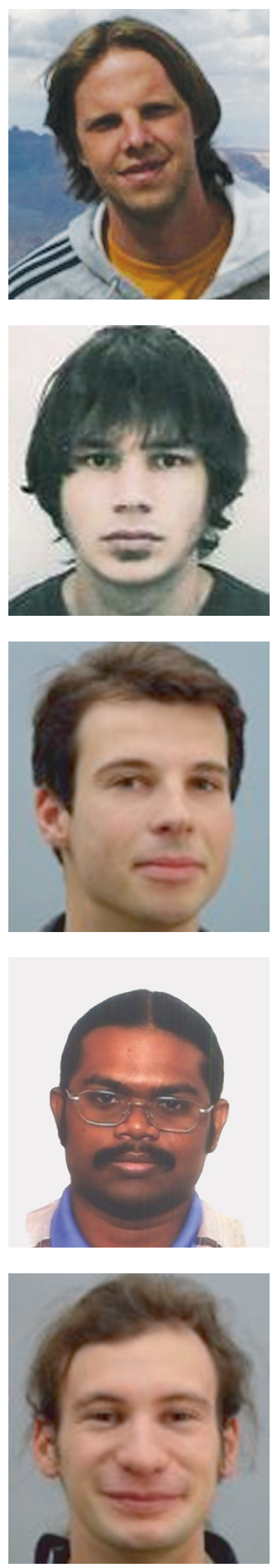

Günther Roelkens (S'02-M'07) received the electronic engineering degree in 2002 and the Ph.D. degree in 2007 from Ghent University, Ghent, Belgium. Since 2002, he has been with the Department of Information Technology, Ghent University-Interuniversity Microelectronics Center (IMEC). Since 2007 he is working as a post-doc in the Photonics Research Group of Ghent University/IMEC. He is also an assistant professor in the Optoelectronic devices group at the Technical University of Eindhoven. His research interests are high efficiency interfaces between optical fiber and photonic integrated circuits and the heterogeneous integration of III-V semiconductors on top of silicon-on-insulator photonic integrated circuits.

Diedrik Vermeulen was born in 1984 in Ghent, Belgium. He graduated in 2006 as a physicist from Ghent University. From 2006 until 2008 he studied engineering (option applied physics) at Ghent University and received the Alcatel-Bell price for his master thesis: "Design and fabrication of a transceiver for Fiber-To-The-Home (FTTH) Optical Networks." Since 2008, he works in the Photonics Research Group of professor Roel Baets at Ghent University under a specialization grant from the IWT (Institute for the promotion of innovation through science and technology in Flanders).

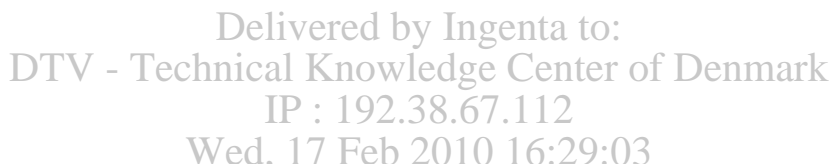

Frederik Van Laere (S'05-M'09) received the degree in electrical engineering in 2004 and the Ph.D. degree in 2009 from Ghent University, Ghent, Belgium. His research interests include the design, fabrication, and characterization of nanophotonic waveguide components and their integration with active functionality.

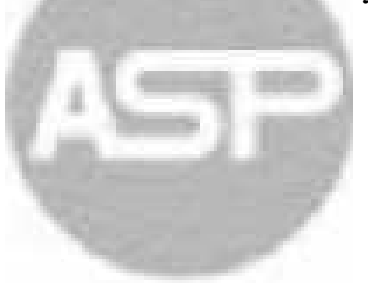

Shankar Selvaraja (S'06) received the M.Tech. degree in Optical communication from College of engineering, Anna University, Chennai, India, in 2004, the M.Sc. degree in Microsystems and Microelectronics from University of Twente, The Netherlands, in 2005. Since 2006 he is working towards his Ph.D. at Photonic research group of IMEC-Ghent University, Belgium. His research interest includes CMOS compatible process development for photonic integrated circuits: optical lithography (193 and $248 \mathrm{~nm}$ ), dry etch and deposition processes. He is a student member of IEEE and IEEE photonics society.

Stijn Scheerlinck (S'04-M'09) received the M.A. degree in electrical engineering in 2003 and the Ph.D. degree in 2009, both from Ghent University, Belgium. His research interests include the modeling and fabrication of metallic grating structures, based on nano-imprint lithography. 

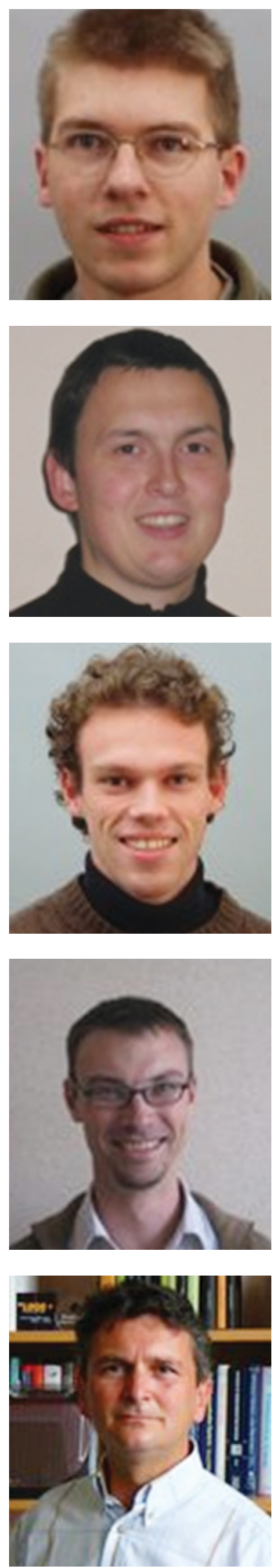

Dirk Taillaert (S'97-M’04) received the M.A. degree in electrical engineering in 1999 and the Ph.D. degree in November 2004, both from Ghent University, Ghent, Belgium. He started working as a Researcher with the Department of Information Technology, Ghent University-Interuniversity Microelectronics Center (IMEC), in August 1999. He has been working on optical sensors with a postdoctoral grant from the Institute for the Promotion of Innovation through Science and Technology (in Flanders). His research interests include the design, fabrication, and characterization of wavelength scale components for photonic integrated circuits.

Wim Bogaerts (S'98-M'05) received the engineering degree in applied physics and the Ph.D. degree from Ghent University, Gent, Belgium, in 1998 and 2004, respectively. He is currently with the Photonics Research Group, Department of Information Technology, Ghent University, Interuniversity Microelectronics Center, where he specialized in the modeling, design, and fabrication of nanophotonic components, particularly photonic crystals. An important focus of his work is the fabrication of SOI photonic nanostructures with deep ultraviolet lithography. Dr. Bogaerts is a member of the IEEE Lasers and Electro-Optics Society and the Optical Society of America.

\section{DTV - Technical Knowledge Center of Denmark}

IP : 192.38 .67 .112

Wed 17 Feb $201016: 29: 03$

Pieter Dumon (S'02-M'07) received the electrical engineering degree in 2002 and the $\mathrm{Ph} . \mathrm{D}$. degree in 2007 from Ghent University, Gent, Belgium. His research interests include the modeling, design, and fabrication of nanophotonic waveguides and structures for passive photonic integrated circuits. Pieter Dumon is the coordinator of EpixFab, the silicon photonics platform.

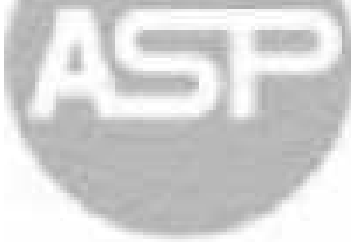

Dries Van Thourhout (S'99-M'00) received the physical engineering degree and the Ph.D. degree from Ghent University, Gent, Belgium, in 1995 and 2000, respectively. He was with Lucent Technologies, Bell Laboratories, Crawford Hill, NJ, from October 2000 to September 2002, working on the design, processing, and characterization of InP/InGaAsP monolithically integrated devices. In October 2002, he joined the Photonics Research Group, Department of Information Technology, Ghent University, Interuniversity Microelectronics Center, continuing his work on integrated optoelectronic devices. His main interests are heterogeneous integration by wafer bonding, intrachip optical interconnect, and wavelengthdivision-multiplexing devices.

Roel Baets (M'88-SM'96) received the electrical engineering degree from Ghent University, Gent, Belgium, in 1980, the M.Sc. degree in electrical engineering from Stanford University, Stanford, CA, in 1981, and the Ph.D. degree from Ghent University, in 1984. He joined the Department of Information Technology (INTEC), Ghent University in 1981, and since 1989, he has been a Professor in the Engineering Faculty. From 1990 to 1994, he has also been a Part-Time Professor at the Technical University of Delft, Delft, The Netherlands. He has mainly worked in the field of photonic components. With about 300 publications and conference papers as well as about ten patents, he has made contributions to the design and fabrication of III-V semiconductor laser diodes, passive guided-wave devices, photonic integrated circuits, and microoptic components. He currently leads the Photonics Research 
Group, INTEC, Ghent University, which is an associated laboratory of the Interuniversity Microelectronics Center, working on integrated photonic devices for optical communication, optical interconnect, and optical sensing. Dr. Baets is a member of the Optical Society of America; the IEEE Lasers and Electro-Optics Society, where he was formerly a Chairman of the Benelux Chapter from 1999 to 2001 and is currently a member of the Board of Governors; the International Society for Optical Engineers; and the Flemish Engineers Association. He has been a member of the program committees of the Optical Fiber Communications Conference, the European Conference on Optical Communication, the IEEE Semiconductor Laser Conference, European Solid-State Device Research Conference, the Conference on Lasers and Electro-Optics-Europe, and the European Conference on Integrated Optics.

testing large scale integrated photonic circuits, comparable to what is done in the micro-electronics world. Therefore, in this paper we will elaborate on another approach to achieve an efficient interface between fiber and nanophotonic integrated circuit, by means of diffractive grating structures. We will show that in this way wafer-scale testing of high index contrast nanophotonic waveguide circuits is feasible and that a high efficiency, broadband interface is realized, which even allows tackling the problem of the huge polarization dependence of high index contrast waveguide structures. The use of diffractive grating couplers will be demonstrated on the silicon-on-insulator high index contrast waveguide platform.

\section{FIBER-TO-CHIP GRATING COUPLER: OPERATION PRINCIPLE AND LIMITATIONS}

In its simplest form, a fiber-to-chip grating coupler consists of a periodic structure defined in a waveguide, realized by etching in a high index waveguide layer or by using deposition techniques. Light impinging on the periodic structure will diffract from the grating under an angle

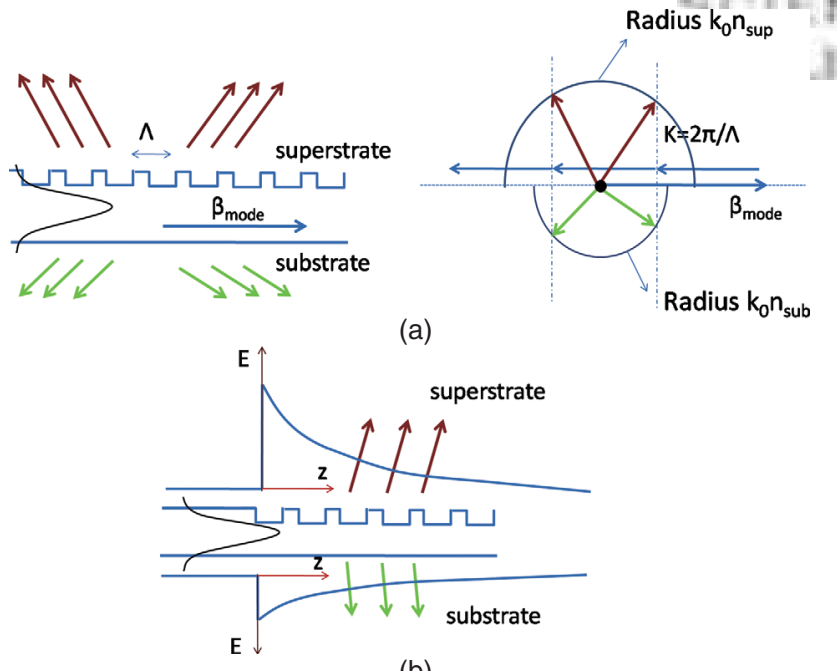

(b)

Fig. 1. Bragg diffraction from a waveguide grating structure to different diffraction orders (a) and the (in first order) exponential decay of the diffracted field strength along the propagation direction of the optical mode (b). determined by the projected Bragg condition, as shown in Figure 1(a). By properly designing the grating structure, diffraction can be limited to a single diffraction order. Placing a single mode fiber under the appropriate angle with respect to the surface of the chip, allows efficient coupling between the optical fiber and the photonic integrated circuit.

In a first order approximation, the field diffracted from a uniform grating structure is exponentially decaying along the propagation direction of light (see Fig. 1(b)). Since efficient interfacing with a single mode fiber is envisaged, the coupling length of the grating (i.e., the $1 / e$ decay length of the diffracted field) needs to be matched with the core size of a single mode fiber, being about $10 \mu \mathrm{m}$. This implies that a strong index contrast in the grating is required, which can easily be obtained in a membranelike waveguide system as silicon-on-insulator. Since the field profile is exponentially decaying, the overlap between the Gaussian mode profile of the optical fiber and the diffracted field is limited, thereby limiting the coupling efficiency between fiber and chip to about $-1 \mathrm{~dB}$, for an optimal grating strength of about $0.14 / \mu \mathrm{m}$ as shown in Figure 2(a). This corresponds to a coupling length of $3.8 \mu \mathrm{m}$. This fiber-to-chip coupling efficiency assumes that all the light from the grating is diffracted towards the optical fiber. A grating coupler based on this design principle for a wavelength band around $1.55 \mu \mathrm{m}$ realized on the SOI waveguide platform is shown in Figure 2(b). It consists of $70 \mathrm{~nm}$ deep grooves on a $630 \mathrm{~nm}$ pitch, etched in a $220 \mathrm{~nm}$ silicon waveguide layer (on a $2 \mu \mathrm{m}$ buried oxide layer). Since high index contrast waveguide structures are used, these one-dimensional grating structures are very polarization dependent and can only be optimized to obtain high performance for a single polarization. Experimentally, $-6 \mathrm{~dB}$ to $-5 \mathrm{~dB}$ coupling efficiency is obtained at a wavelength of $1.55 \mu \mathrm{m}$ from these structures, for transverse electric (TE) polarized light when tilting the fiber 10 degrees off vertical. This is significantly lower than the theoretical $-1 \mathrm{~dB}$ limit. This is due to the fact that in these classical grating coupler structures, also a considerable amount of light is diffracted towards the substrate as shown in Figure 3, limiting the directionality $D$ of the grating (being the ratio of the power diffracted towards the optical fiber and the total power diffracted from the grating structure). Thereby the fiberchip coupling efficiency $\eta$ is limited to $\eta=0.8 D$. Since 


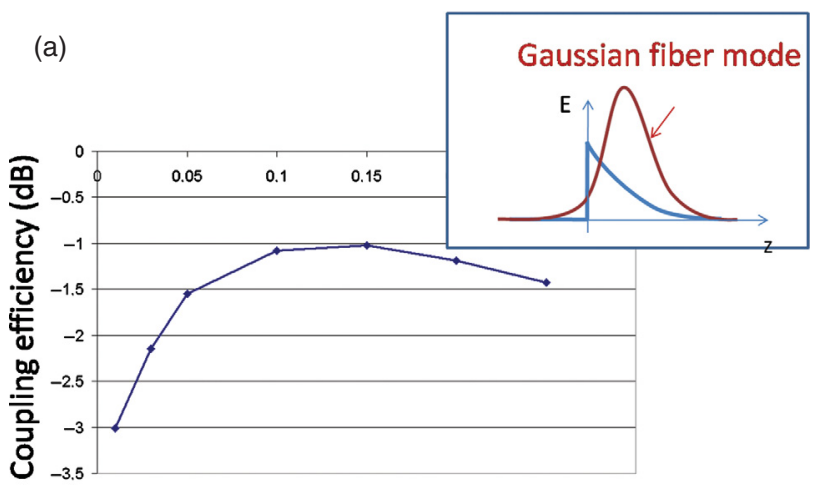

Grating strength $(1 / \mu \mathrm{m})$

(b)

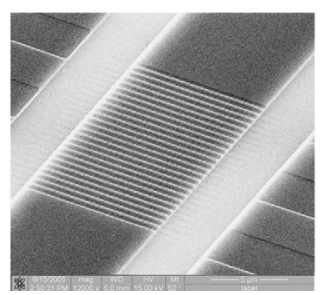

Fig. 2. Influence of the grating strength on the maximum obtainable fiber coupling efficiency, due to the overlap between the Gaussian fiber mode and the exponentially decaying diffracted field profile (a) and an SEM image of a realized uniform diffraction grating, etched $70 \mathrm{~nm}$ in a $220 \mathrm{~nm}$ Silicon-on-Insulator waveguide structure (b).

the directionality for basic grating coupler structures is about $50 \%$, the fiber-chip coupling loss is mainly related to this limited directionality. Therefore, in the remainder of this section we will primarily focus on the design, fabrication and characterization of highly directional diffraction gratings. A further improvement in fiber coupling efficiency can be obtained by using non-uniform diffraction gratings in order to better match the diffracted field profile to that of the Gaussian fiber mode. ${ }^{2}$ This however substantially complicates fabrication, since nanometer-scale dimensional control is required over each individual grating line, making the definition of the grating structures using CMOS compatible deep UV lithography hard. Due to the strong polarization dependence of high index contrast structures, the devices described in the remainder of this section were only optimized and characterized for TE

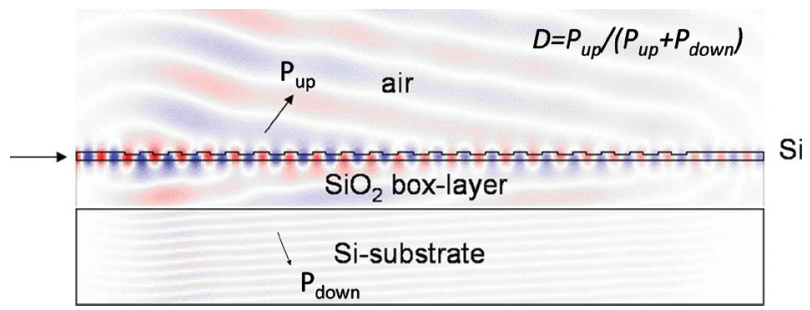

Fig. 3. Field plot of the diffraction of light from a uniform grating structure showing the limited directionality of basic grating structures which limits the fiber-to-chip coupling efficiency. polarized light. The realization of polarization independent fiber-chip coupling will be discussed later on in this paper.

High directionality gratings can be obtained in a number of ways, schematically outlined in Figure 4. One way to achieve high directionality is to integrate a bottom mirror underneath the diffractive grating structure. This can be a metallic mirror ${ }^{3}$ or a dielectric DBR mirror. ${ }^{4}$ Even the use of a high index substrate as a carrier for the membrane waveguide structures (as is the case for a siliconon-insulator waveguide structure) can already positively impact the fiber coupling efficiency. The mirror redirects the downwards diffracted light towards the optical fiber,

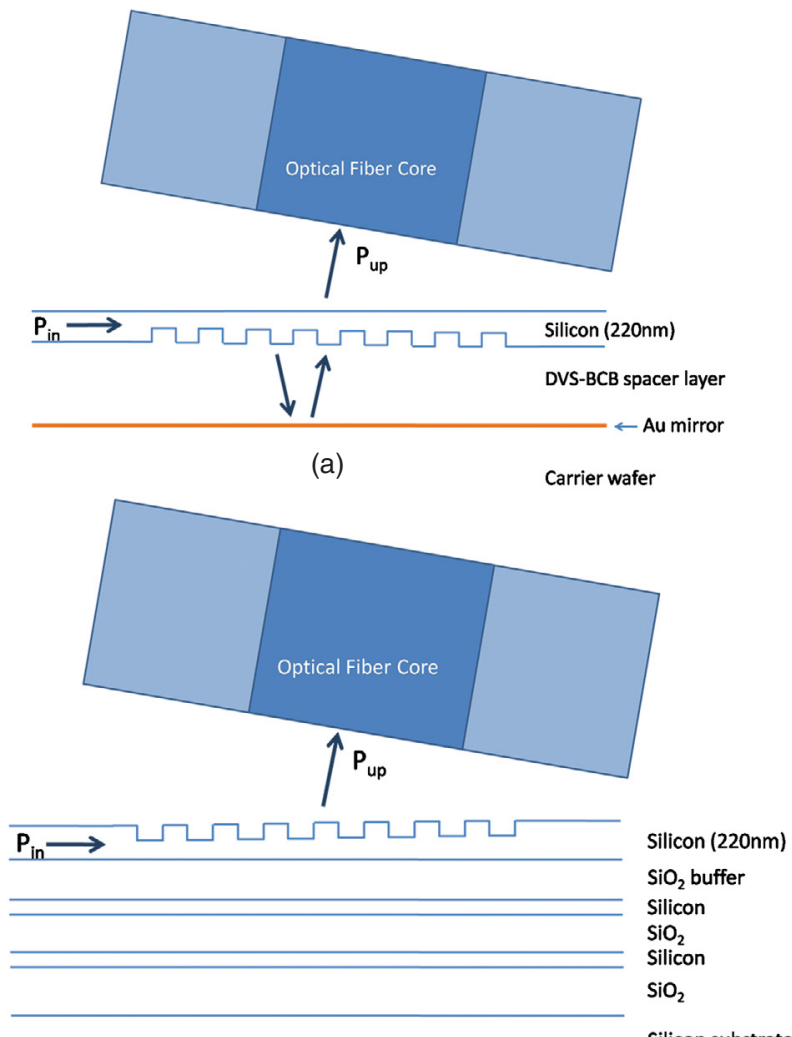

(b)

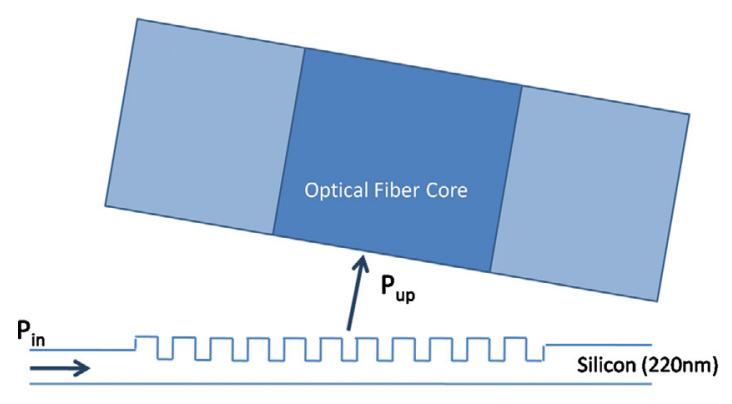

$\mathrm{SiO}_{2}$ buffer layer (2000nm)

(c)

Silicon substrate

Fig. 4. Highly directional diffractive grating structures based on a metallic bottom mirror (a), a dielectric DBR mirror (b) and a silicon overlay (c). 
where it interferes with the directly upwards diffracted beam. Since this is an interference based enhancement of the grating directionality, the phase of the redirected beam needs to be well controlled, since it will determine the coupling strength of the grating/mirror combination and hence the coupling efficiency to optical fiber. This is illustrated in Figure 5, where the influence of the thickness of the buried oxide, separating the silicon waveguide layer from the silicon substrate, on the fiber coupling efficiency of the basic SOI grating coupler (shown in Fig. 2(b)) is depicted. This buried oxide thickness determines the phase of the redirected beam and thereby the fiber coupling efficiency. Experimentally we have realized high efficiency one-dimensional grating structures based on a gold bottom mirror and using a two-pair $\mathrm{Si} / \mathrm{SiO}_{2}$ DBR stack. The gold bottom mirror based devices were realized using a DVS-BCB wafer bonding approach. In this case the basic grating structures with limited directionality shown in Figure 2(b) were used. On top of these diffraction gratings a DVS-BCB spácer layer $(n \neq 1.54)$ of $840 \mathrm{~nm}$ and a gold mirror of $50 \mathrm{~nm}$ were defined, after which the whole device structure was bonded $/$ on a carrier wafer and the original silicon carrier wafer was removed in order to access the fiber couplers. The fabricated structures are shown in Figure 6(a). In this way $-1.6 \mathrm{~dB}$ coupling efficiency was obtained. While these grating structures based on metallic bottom mirrors allow for high efficiency fiber coupling, the fabrication process is not directly transferable to a CMOS line, since it requires the use of non-standard bonding processes. Therefore a $\mathrm{Si} / \mathrm{SiO}_{2}$ DBR based approach was pursued since it allows for fabrication in a CMOS line using standard processes. The device structure is shown in Figure 6(b). The fiber couplers were fabricated on a $200 \mathrm{~mm} \mathrm{Si}$ wafer. First a $1 \mu \mathrm{m} \mathrm{SiO}{ }_{2}$ isolation layer is deposited on a bare Si wafer. Then the Bragg mirror, consisting of two $112 \mathrm{~nm}$ layers of amorphous silicon and a $267 \mathrm{~nm}$ layer of $\mathrm{SiO}_{2}$ are deposited. On top of the Bragg mirror a $1.48 \mu \mathrm{m}$ of buffer

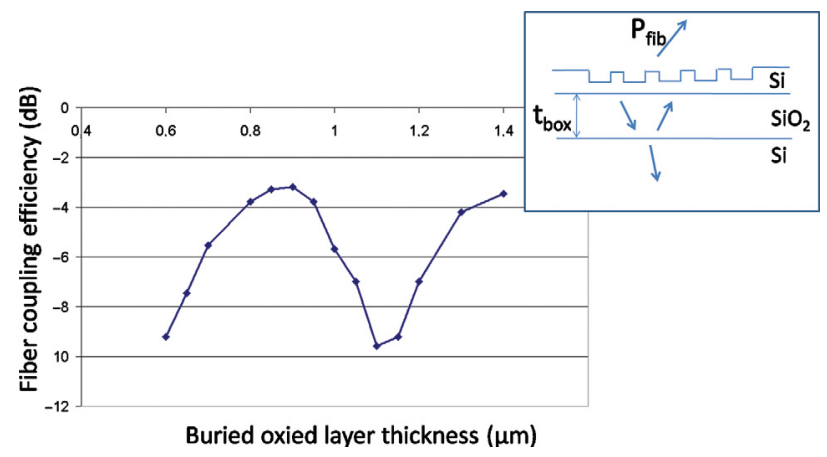

Fig. 5. Simulation of the influence of the buried oxide layer thickness $t_{\text {box }}$ on the fiber-to-chip coupling efficiency at a wavelength of $1.55 \mu \mathrm{m}$ (TE polarization). The grating period is $630 \mathrm{~nm}$, the grating is etched $70 \mathrm{~nm}$ in the $220 \mathrm{~nm}$ silicon waveguide layer and the fiber is tilted 10 degrees from the surface normal.
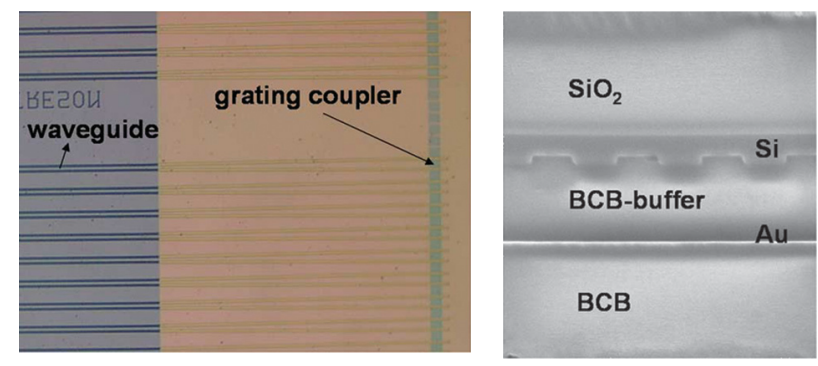

(a)

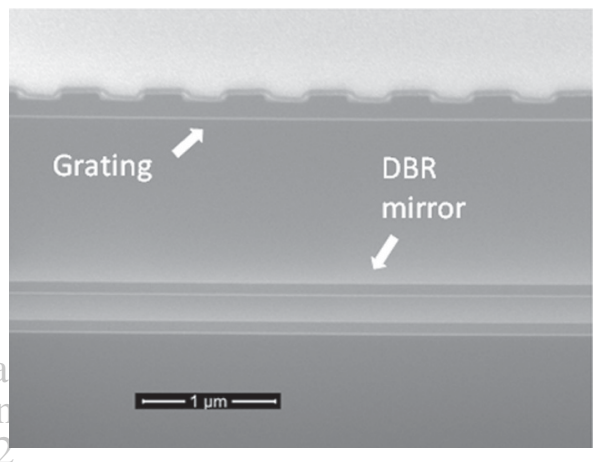

(b)

Fig. 6. Experimental realizations of high efficiency fiber-to-chip coupling structures: A gold bottom mirror grating structure (a). Reprinted with permission from [3], F. Van Laere et al., J. Lightwave Technology 25, 151 (2007). (C) 2007, IEEE, and a $\mathrm{Si} / \mathrm{SiO}_{2}$ DBR bottom mirror grating structure (b). The fiber-to-chip coupling efficiency is $-1.6 \mathrm{~dB}$ for both structures.

$\mathrm{SiO}_{2}$ and a silicon layer were deposited. The grating couplers and waveguides were fabricated using $193 \mathrm{~nm}$ optical lithography and dry etching. Again, the basic grating structure was used, in order to compare the metallic mirror and DBR mirror approach. The experimentally obtained coupling efficiency is comparable to the metallic mirror approach, illustrating that in an all-dielectric approach also high efficiency coupling structures can be realized.

While the use of a bottom mirror significantly increases the fiber-chip coupling efficiency, it requires dedicated substrates or dedicated processing steps. Intrinsically improving the directionality of the grating structures would allow realizing high efficiency fiber-to-chip coupling independent of the substrate. By optimizing the design of the diffraction grating, this can be achieved. The use of blazed gratings allows increasing the directionality of the grating structure, but these structures are not compatible with CMOS fabrication. In Figure 4(c), a grating coupler structure with a silicon overlay is presented to realize high directionality gratings. ${ }^{5}$ The silicon overlay gives an additional degree of freedom to design the grating structure, such that it intrinsically has a high directionality and a coupling strength that is well matched to interface with a single mode fiber. By optimizing the design of this grating structure, $-1 \mathrm{~dB}$ coupling efficiency can be obtained for a uniform grating structure on a standard SOI waveguide 
wafer, almost independent of the buried oxide layer thickness. This silicon overlay can be a deposited amorphous layer or epitaxially grown silicon. A prototype grating structure with epitaxially grown silicon overlay is shown in Figure 7(a). The fabricated structure consists of a uniform grating structure with a pitch of $610 \mathrm{~nm}$ (duty cycle of $50 \%)$. This grating is etched in a silicon layer stack of $400 \mathrm{~nm}$ height $(220 \mathrm{~nm}$ silicon waveguide layer with a $180 \mathrm{~nm}$ thick silicon overlay epitaxially grown on top) by etching $250 \mathrm{~nm}$ deep slits. While the grating parameters of the fabricated grating structure deviated from the designed ones $(150 \mathrm{~nm}$ overlay thickness and $220 \mathrm{~nm}$ etch depth were targeted), already $-2.6 \mathrm{~dB}$ fiber coupling efficiency was realized in this way, by tilting the fiber 10 degrees off vertical, which is in good agreement with FDTD simulation results. ${ }^{6}$ This good correspondence between measurement and simulation makes us confident that in future optimized fabrication runs $-1 \mathrm{~dB}$ coupling efficiency is achievable.

While a high fiber-to-chip coupling efficiency is an important issue, the optical bandwidth of the diffraction grating is equally important for practical applications. Diffraction grating structures are typically considered to be narrow band structures, due to the strong wavelength dependence of the diffraction angle on the wavelength. This is however only the case for low index contrast gratings, where the grating length is on the order of several tens to hundreds of wavelengths. Since high index contrast grating structures are used to interface with a single mode

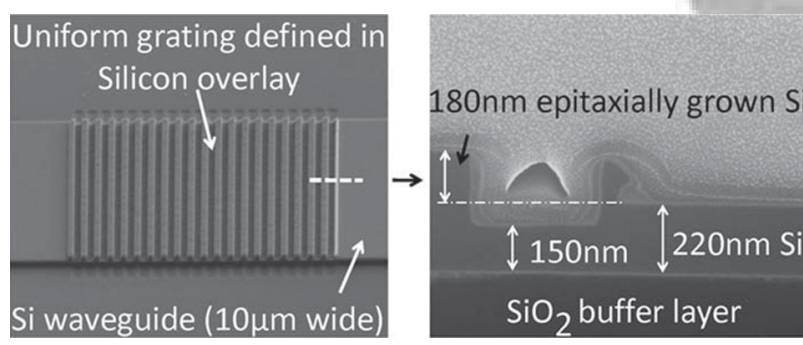

(a)

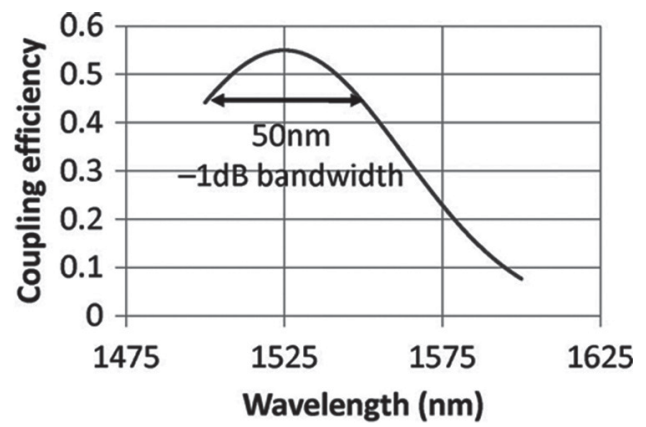

(b)

Fig. 7. SEM image of a fabricated grating structure with a silicon overlay, defined using silicon epitaxial growth (a) and the measured fiberto-chip coupling spectrum (b) which is in good agreement with FDTD simulations. Reprinted with permission from [6], G. Roelkens et al., Appl. Phys. Lett. 92, 131101 (2008). (C) 2008, American Institute of Physics. fiber, this also brings along a large optical bandwidth. Typically a $1 \mathrm{~dB}(3 \mathrm{~dB})$ bandwidth of $30 \mathrm{~nm}$ to $50 \mathrm{~nm}(50 \mathrm{~nm}$ to $100 \mathrm{~nm}$ ) is realized, depending on the device structure as shown in Figure 7(b).

\section{FOCUSING GRATING COUPLERS}

While the diffraction grating used to interface with the optical fiber is very compact, i.e. about the size of the core of a single mode fiber, still a long adiabatic taper is required to laterally transform the $10 \mu \mathrm{m}$ wide waveguide to a $0.5 \mu \mathrm{m}$ wide single mode waveguide. This adiabatic taper consumes significant silicon real estate, which becomes an important issue in large scale integrated optical circuits. Therefore, focusing grating structures were designed to replace the adiabatic taper. ${ }^{7}$ The grating lines
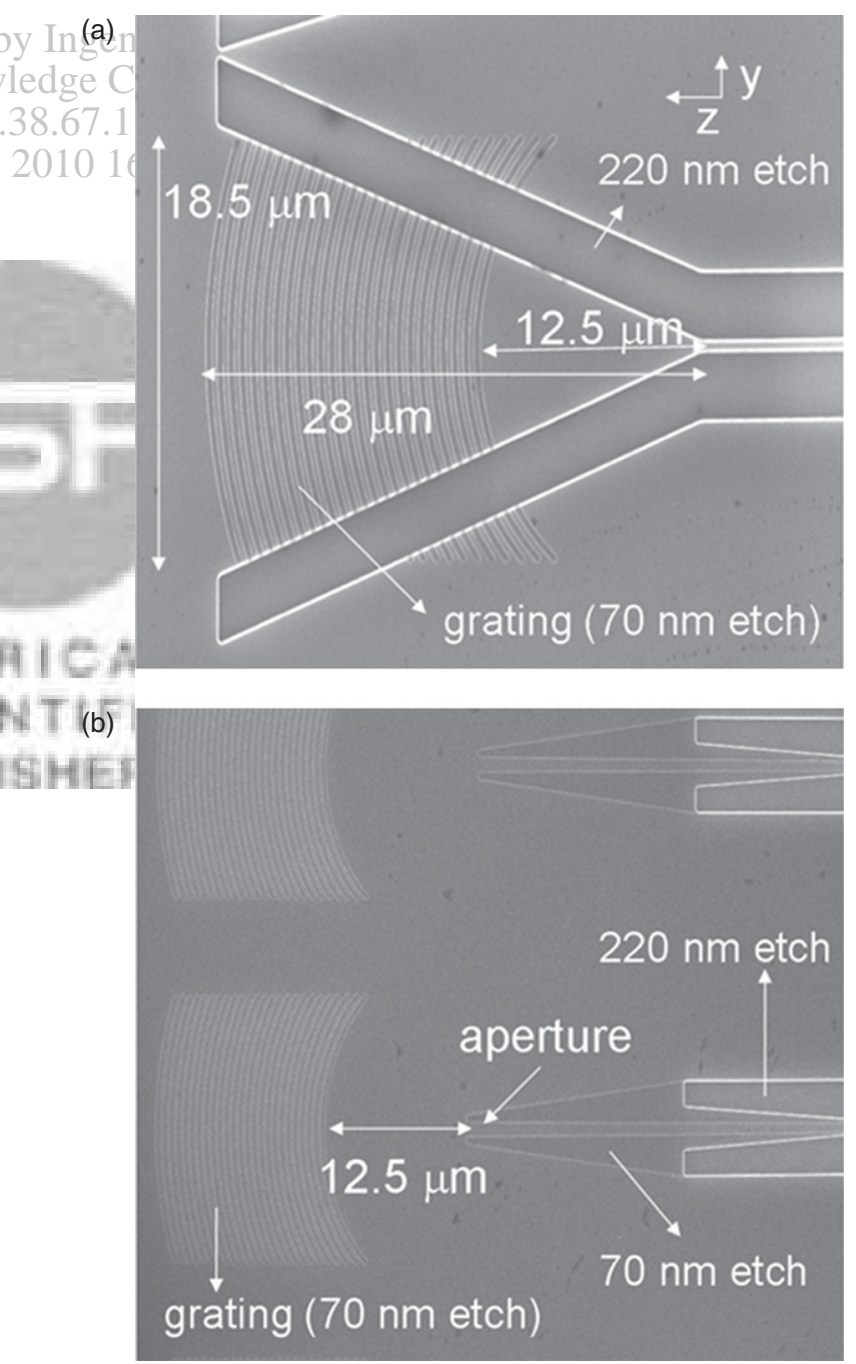

Fig. 8. Two implementations of a focusing grating coupler: A focusing grating in combination with a short, linear, non-adiabatic taper (a) and in (b) the light is focused by the grating onto a low contrast aperture. Reprinted with permission from [7], F. Van Laere et al., Group IV Photonics, paper ThB3 (2008). (C) 2007, IEEE. 
of the focusing grating are parts of ellipses with a common focal point. At the common focal point the single mode waveguide is positioned. On the symmetry axis of the focusing grating coupler the pitch between the grating lines is $630 \mathrm{~nm}$, as in the basic rectilinear grating configuration for TE polarized light at a wavelength of $1.55 \mu \mathrm{m}$. The fiber is tilted 10 degrees off vertical. These structures allow realizing an eight-fold decrease in the length of the grating coupling structure, without having an impact on the device efficiency. The realized focusing grating coupler structures are shown in Figure 8 in two different configurations. In a first implementation, a focusing grating in combination with a short, linear, non-adiabatic taper to a $500 \mathrm{~nm}$ wide photonic wire is used. In a second implementation, the light is focused by the grating onto a low contrast aperture with a width ranging from 0.8 to $2 \mu \mathrm{m}$. This low-contrast aperture was implemented in a $70 \mathrm{~nm}$ etch (instead of being fully etched through the $220 \mathrm{~nm}$ silicon waveguide layer) using the same etch step used to define the grating lines. The lower lateral index contrast reduces reflections at the aperture. The transition from low-contrast to high-contrast waveguides is done adiabatically over a $30 \mu \mathrm{m}$ length. The performance of both types of focusing grating couplers is comparable to the basic non-focusing structures: $-6 \mathrm{~dB}$ to $-7 \mathrm{~dB}$ coupling efficiency can be obtained.

\section{EXTENDING THE BANDWIDTH: GRATING DUPLEXER STRUCTURES}

For a set of applications, the bandwidth provided by the diffraction grating structures described above is insufficient. This is especially true for applications requiring the processing of multiple wavelength bands on the photonic integrated circuit. In these applications, the photonic integrated circuit typically has to interface with the optical fiber in two well separated wavelength bands, which have to be (de)multiplexed on the photonic integrated circuit. This is for example the case for fiber-to-thehome (FTTH) access network transceivers which require the multiplexing of a $1300 \mathrm{~nm}$ wavelength band signal and a $1490 \mathrm{~nm} / 1550 \mathrm{~nm}$ wavelength band signal. ${ }^{8}$ While the basic grating structures as discussed in the previous section do not provide sufficient bandwidth for this task, the design of the grating structure can be modified in order to accommodate the efficient coupling of two wavelength bands, while at the same time providing the wavelength duplexing functionality. This can be achieved by using the two access waveguides to the grating as shown in Figure 9(a) and designing the grating period and fiber angle such that both wavelength bands are coupled in opposite directions on the photonic integrated circuit. The same mechanisms as described in the previous section to enhance the fiber coupling efficiency can be used to improve the performance of this device. In Figure 9(b), the (a)
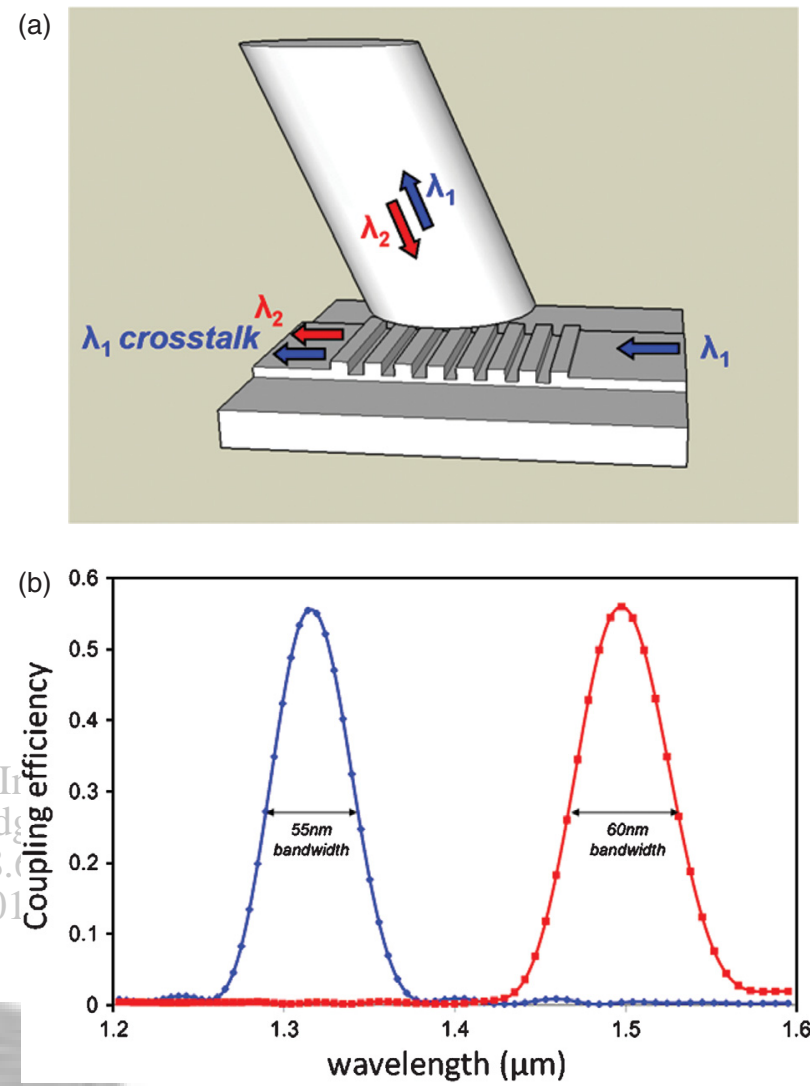

Fig. 9. The grating duplexer concept: device layout (a) and simulation of the performance of this one dimensional grating duplexer structure with silicon overlay for TE polarized light (b). Reprinted with permission from [8], G. Roelkens et al., Opt. Express 15, 10091 (2007). (C) 2007, Optical Society of America.

simulation results are plotted for an optimized duplexer grating /structure for $1310 \mathrm{~nm}$ and $1490 \mathrm{~nm}$ wavelength based on a silicon overlay grating structure. $-2.6 \mathrm{~dB}$ coupling efficiency can be obtained for both wavelength bands, while the $3 \mathrm{~dB}$ bandwidth for the individual bands is $55 \mathrm{~nm}$ and $60 \mathrm{~nm}$ respectively. The measurement results of a prototype duplexing structure realized by etching $70 \mathrm{~nm}$ deep slits in the $220 \mathrm{~nm}$ silicon waveguide layer thickness are shown in Figure 10(a). The period of the grating is $520 \mathrm{~nm}$ and the grating duty cycle is $40 \%$. The grating consists of 20 grating periods. Index matching fluid was applied between the optical fiber facet and the grating duplexer to avoid reflections at the fiber facet and the fiber was tilted under an angle of 10 degrees. Transverse electric polarization was used. The duplexing behavior can clearly be observed. When using this component in a transceiver configuration, where one wavelength band is used for upstream communication, while the other wavelength band is used for downstream communication, the crosstalk from the transmitter port to the receiver port has to be minimized. While the intrinsic grating duplexer has a significant crosstalk of about $-10 \mathrm{~dB}$, this can circumvented by implementing an optical filter (e.g., an echelle 


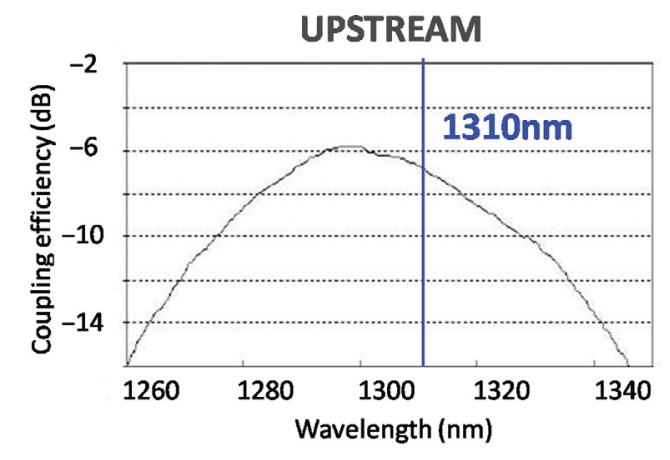

(a)

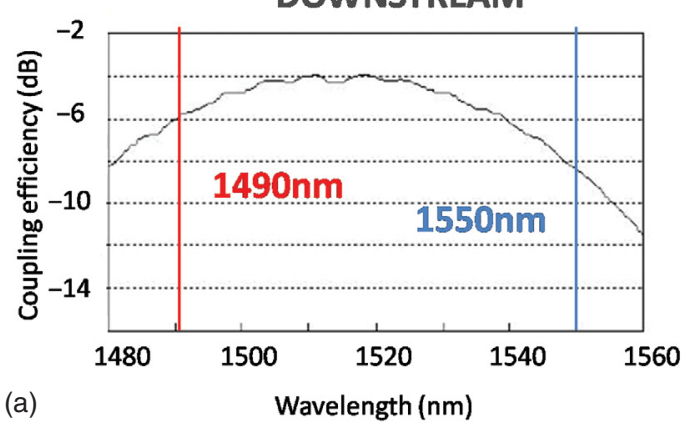

$1490 \mathrm{~nm} / 1550 \mathrm{~nm} \quad 1310 \mathrm{~nm}$

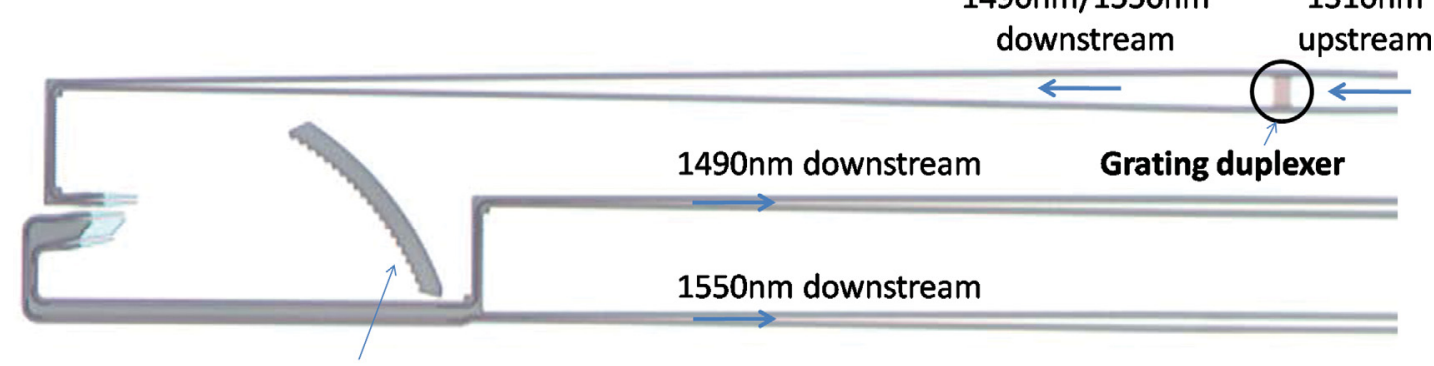

planar concave grating

(b)

Fig. 10. Measured performance of a one-dimensional grating duplexer by directly etching the grating in the $220 \mathrm{~nm}$ silicon waveguide layer (a) and the use of an echelle grating structure to reduce the crosstalk levels between the upstream and downstream channels below $-30 \mathrm{~dB}$.

grating structure) in the downstream path, as shown in Figure 10(b). The measured residual crosstalk between the transmitter port and the receiver in this device was better than $-30 \mathrm{~dB} .^{9}$

\section{TWO-DIMENSIONAL GRATING STRUCTURES-A POLARIZATION DIVERSITY SCHEME}

The use of high refractive index contrast waveguide structures implies that the photonic integrated circuit behaves very differently for transverse electric (TE) and transverse magnetic (TM) polarized light. This is also the case for the one-dimensional fiber-to-chip grating coupler structures discussed in the previous sections. For practical applications often polarization independent operation of the photonic integrated circuit is required, since the polarization of the light in the optical fiber is unknown and varying over time. The use of two-dimensional grating structures allows tackling this problem using a polarization diversity configuration. This is schematically depicted in Figure 11(a). The two-dimensional grating structure can be seen as the superposition of two one-dimensional grating structures, which are identical and which are designed to efficiently couple a single polarization (i.e., TE polarized light) to the waveguide circuit. By placing these one-dimensional grating structures orthogonal to each other, hence creating a square lattice grating structure, this two-dimensional grating structure allows to efficiently interface with both polarizations of light in the optical fiber, by coupling the orthogonal polarizations in the optical fiber to identically (TE) polarized modes in orthogonal waveguides. This approach allows realizing polarization independent operation of (intrinsically very polarization dependent) high index contrast waveguide structures by having two identical photonic integrated circuits in both arms of the polarization diversity configuration as shown in Figure 11(b). While achieving "identical" circuits requires sub-nanometer control over the dimensions of the photonic integrated circuit waveguides, this can be achieved using state-of-the-art $193 \mathrm{~nm}$ deep UV lithography. The operation principle of the twodimensional grating structure as described above is only valid when the fiber is perfectly perpendicular to the photonic integrated circuit. While a diffraction grating can be designed to diffract light perfectly perpendicular to the chip surface, this is always associated with a strong second order Bragg reflection from the grating structure, which is not wanted for practical circuits. Therefore the fiber needs to be slightly tilted with respect to the surface normal and directed along the symmetry axis of the twodimensional grating structure. While this allows avoiding the second order reflection, it also requires that the optical waveguides are slightly tilted inwards, as is clear from the projected Bragg condition as shown in Figure 12(a). The basic two dimensional grating structure consists of a square lattice of holes (diameter of $390 \mathrm{~nm}$ ) on a pitch of $605 \mathrm{~nm}$, which are etched $70 \mathrm{~nm}$ deep in the silicon waveguide layer. The fiber is tilted 10 degrees off vertical to avoid second order Bragg reflection. Therefore the optical 


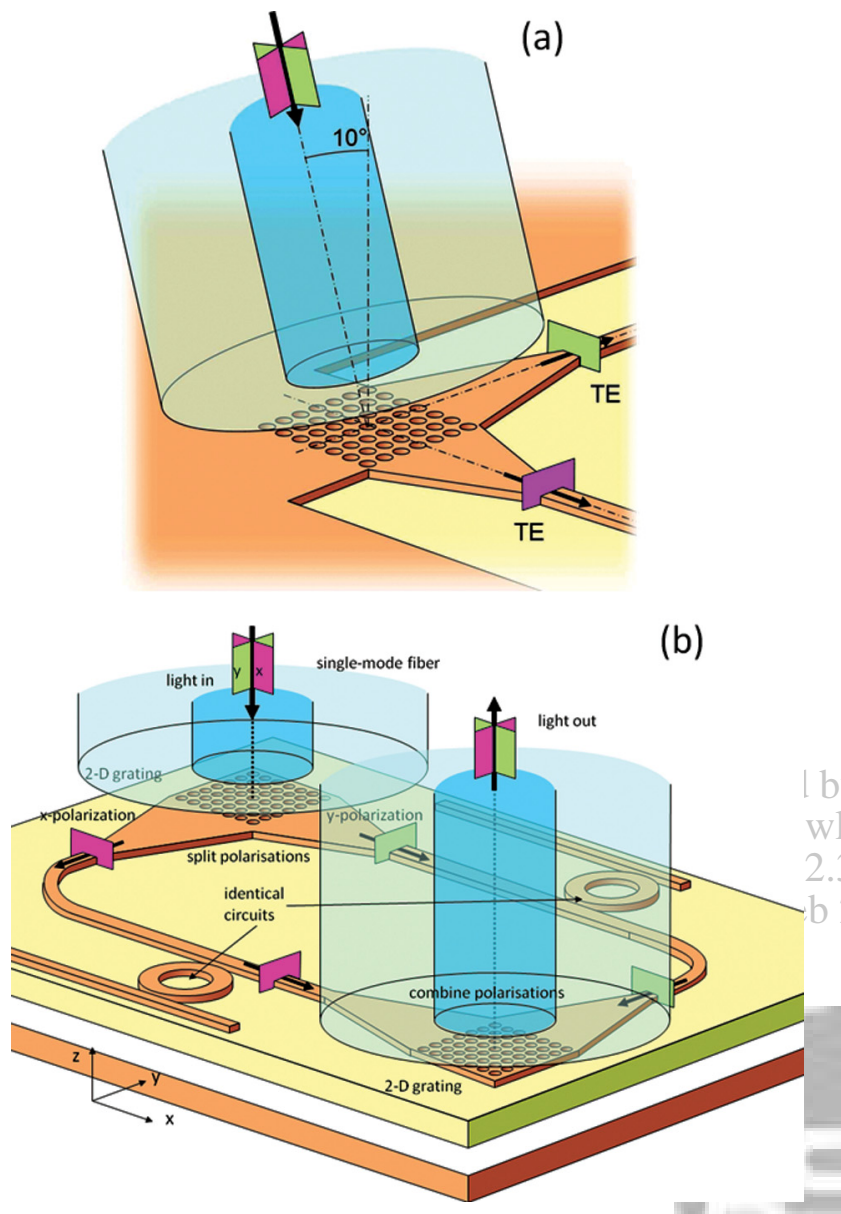

Fig. 11. Operation principle of a two-dimensional grating structure (a) and using this two-dimensional grating structure in a polarization diversity configuration (b) to obtain polarization insensitive operation of high index contrast waveguide structures.

access waveguides are tilted 3.1 degrees inwards. In practice the tilting of the optical fiber off vertical implies that the polarization independent behavior only works over a limited wavelength range, as shown in Figure 12(b), where the measured wavelength dependence of the polarization dependent loss of a two-dimensional grating structure is plotted. The polarization dependent loss can be kept below $1 \mathrm{~dB}$ over a wavelength range of $15 \mathrm{~nm}$.

While the two-dimensional grating structure is an elegant way of solving both the fiber-chip coupling problem and the polarization dependence of photonic integrated circuits, the limited bandwidth over which low polarization dependence is obtained can be an issue in practical applications. One way to tackle the problem is to improve the design of the grating structure (shape of the holes, grating lattice structure) to obtain better polarization insensitivity. This however requires extensive three-dimensional FDTD simulations which require a lot of computational resources. At the moment it is not yet clear what the ultimate limit of the performance of this type of devices is in terms of polarization dependent loss. (a)

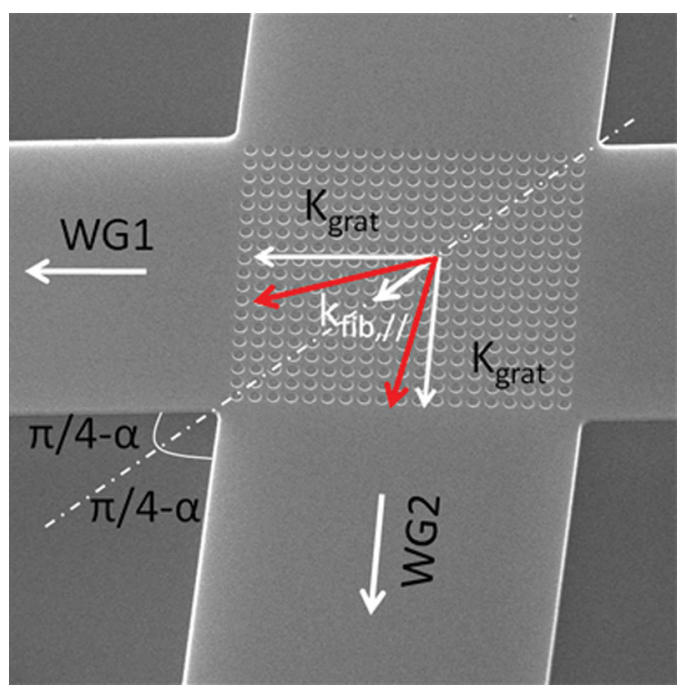

(b)

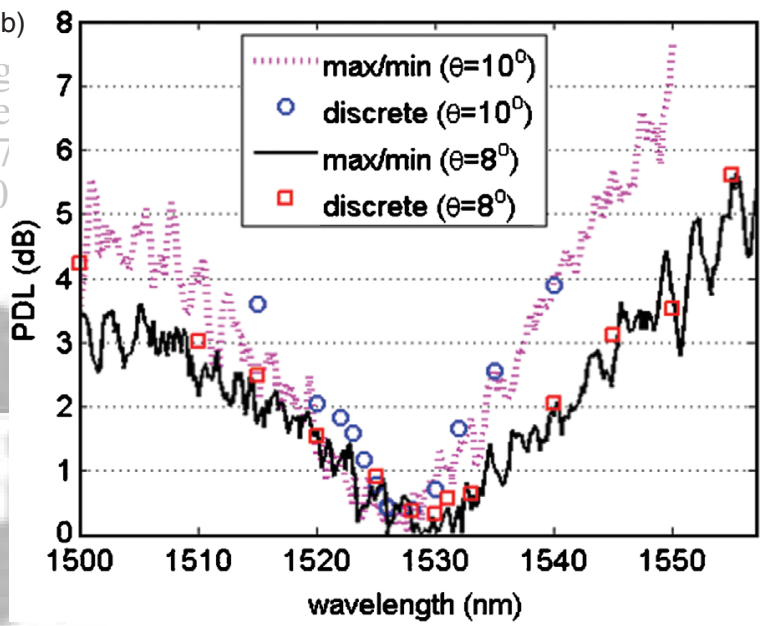

Fig. 12. Influence of the tilt of the optical fiber along the symmetry axis of the two-dimensional grating on the orientation of the access waveguides (a) and wavelength dependence of the polarization dependent loss (PDL) due to the tilting of the optical fiber (b).

Since the two-dimensional grating structures can be considered as a superposition of two one-dimensional grating structures, it is relatively straightforward to also design a focusing polarization diversity grating structure. This allows realizing a polarization agnostic interface between the optical fiber and the photonic integrated circuit on a drastically scaled silicon real estate area as shown in Figure 13. The performance of the device in terms of efficiency and polarization dependent loss is comparable to that of the standard two-dimensional grating structures. ${ }^{10}$

\section{THE OPTICAL PROBE}

As discussed above, diffractive grating structures allow efficient interfacing with an optical fiber. Since they do not require a polished facet, wafer scale testing of the complete photonic integrated circuit can be done, using the circuit input/output grating couplers as the testing sites. 


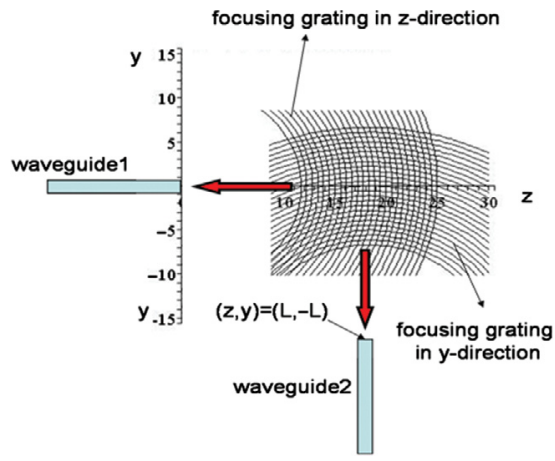

(a)
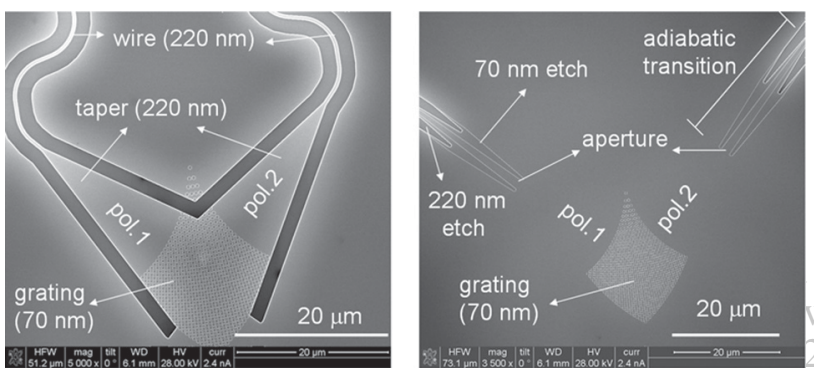

(b)

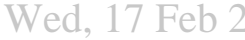

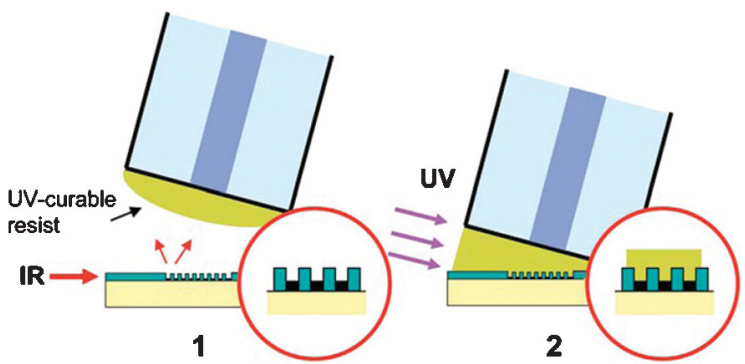
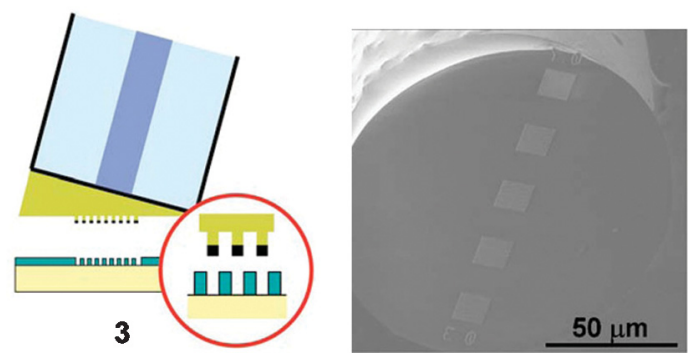

Fig. 14. Schematic of the fabrication process for the optical probe based on nano-imprint lithography and an SEM image of a fabricated optical probe. Only the center grating covers the core of the single mode fiber. 3 Reprinted with permission from [11], S. Scheerlinck et al., Appl. Phys. Lett. 92,031104(2008). (C) 2008, American Institute of Physics.
Fig. 13. Schematic of the construction of a two-dimensional focusing grating coupler as the superposition of two one-dimensional diffraction gratings (a) and the experimental realization of these focusing grating structures in two implementations, as in the case of the one-dimensional focusing grating coupler (b). Reprinted with permission from [10], F. Van Laere et al., Focusing Polarization Diversity Grating Couplers in Siliconon-Insulator, accepted for publication in Journal of Lightwave Technology. (C) 2009, IEEE.

This approach however does not allow testing of the individual components on the photonic integrated circuit, since implementing grating couplers at the input and output of the individual components would prevent the complete circuit from working. Testing individual components requires some sort of optical probe, analogous to the electrical probes commonly used in micro-electronics, which allow accessing the pads of individual components on an integrated circuit. These optical probes can be realized by defining a diffraction grating not on the waveguide circuit but on the facet of the optical fiber. ${ }^{11}$ In Figure 14 the method to define this grating structure is outlined. A gold grating was defined on a fiber surface using a nano-imprint technique. First, the fiber with UV-curable resist is aligned over the specially prepared mold carrying the 10 by $10 \mu \mathrm{m}$ gold grating pattern in the trenches of the mold. Upon attachment, the cavities are filled and the resist is UV cured. Finally, the mold is released. The metal grating is now attached to the fiber, due to the fact that the mold was covered with an anti-stiction film prior to gold deposition. By using gold as the grating material, a high refractive index contrast is obtained. A fabricated optical probe is shown in Figure 14. This way efficient coupling between the optical probe and the photonic integrated circuit can be obtained. Experimentally $15 \%$ coupling efficiency between a single mode fiber probe and a $3 \mu \mathrm{m}$ wide photonic integrated circuit was realized for TE polarized light at $1.55 \mu \mathrm{m}$ wavelength. The grating consisted of $20 \mathrm{~nm}$ thick gold stripes on a $630 \mathrm{~nm}$ pitch. The realization of this optical probe can pave the way to wafer-scale testing of high index contrast large scale integrated waveguide circuits.

\section{CONCLUSIONS}

Photonic integrated circuits have tremendous benefits over their discrete counterparts. Especially the use of high index contrast waveguide structures for photonic integrated circuits allows large scale integration of optical functions on a single substrate, reducing the cost, size and weight of the devices, while improving their performance. The interfacing of these scaled optical circuits with an optical fiber and the wafer-scale testing of the integrated circuits is one of the most important aspects for bringing high index contrast waveguide structures from research to practical applications. In this paper we have outlined the use of diffraction gratings to achieve this fiber/chip interfacing and wafer-scale testing. We have shown that high efficiency coupling can be achieved and that the problem of the huge polarization dependence of high index contrast waveguide systems can be circumvented by using a two-dimensional grating coupler approach. The extension of the bandwidth of the grating couplers for applications which require the fiber-chip coupling of two wavelength bands is described. Finally, in order to go to real waferscale testing of photonic integrated circuits, an optical 
probe is presented which allows probing each individual component of the photonic integrated circuit.

Acknowledgments: G. Roelkens acknowledges Scientific Research-Flanders (FWO) for a postdoctoral grant. This work is supported partially by EU-funded projects FP7-HELIOS, FP6-ePIXnet and IWT-SBO epSOC project.

\section{References and Notes}

1. S. McNab, N. Moll, and Y. Vlasov, Opt. Express 11, 2927 (2003).

2. D. Taillaert, P. Bienstman, and R. Baets, Opt. Lett. 29, 2749 (2004).

3. F. Van Laere, G. Roelkens, M. Ayre, J. Schrauwen, D. Taillaert, D. Van Thourhout, T. Krauss, and R. Baets, J. Lightwave Technology 25,151 (2007).

4. S. Selvaraja, D. Vermeulen, M. Schaekers, E. Sleeckx, W. Bogaerts, G. Roelkens, P. Dumon, D. Van Thourhout, and R. Baets, Highly efficient grating coupler between optical fiber and silicon photonic circuit, Conference on Lasers and Electro-Optics (2009), p. CTuC6.

5. G. Roelkens, D. Van Thourhout, and R. Baets, Opt. Express 14, 11622 (2006).

6. G. Roelkens, D. Vermeulen, D. Van Thourhout, R. Baets, S. Brision, P. Lyan, P. Gautier, and J. M. Fedeli, Appl. Phys. Lett. 92, 131101 (2008).

7. F. Van Laere, W. Bogaerts, P. Dumon, G. Roelkens, D. Van Thourhout, and R. Baets, Group IV Photonics, paper ThB3 (2008).

8. G. Roelkens, D. Van Thourhout, and R. Baets, Opt. Express 15, 10091 (2007).

9. D. Vermeulen, G. Roelkens, J. Brouckaert, D. Van Thourhout, R. Baets, R. Duijn, E. Pluk, and G. Van den Hoven, European Conference on Optical Communication ECOC, Belgium (2008), p.Tu.3.C.6.

10. F. Van Laere, W. Bogaerts, P. Dumon, G. Roelkens, D. Van Thourhout, and R. Baets, Focusing Polarization Diversity Grating Couplers in Silicon-on-Insulator, accepted for publication in Journal of Lightwave Technology.

11. S. Scheerlinck, D. Taillaert, D. Van Thourhout, and R. Baets, Appl. Phys. Lett. 92, 031104 (2008).

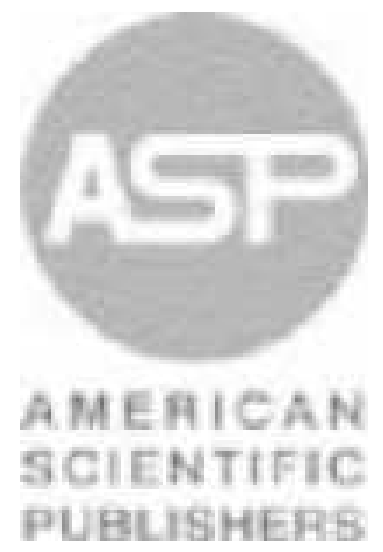

